\title{
[Si/Ti] elemental ratios in 9 old halo globular clusters
}

\author{
Jae-Woo Lee ${ }^{1}$ and Bruce W. Carney ${ }^{2}$ \\ ${ }^{1}$ Astrophysical Research Center for the Structure and Evolution of the Cosmos, \\ Department of Astronomy and Space Science, Sejong University, \\ 98 Gunja-Dong, Gwangjin-Gu, Seoul, 143-747, Korea \\ email: jaewoolee@sejong.ac.kr \\ ${ }^{2}$ Department of Physics and Astronomy, University of North Carolina, \\ Chapel Hill, NC 27599-3255, USA \\ email: bruce@physics.unc.edu
}

\begin{abstract}
We present elemental abundance ratios for red giant stars in 9 old halo globular clusters based on the homogeneous data sample and analysis method. Our result suggests that [Si/Ti] ratios in old globular clusters decrease with Galactocentric distances, confirming the previous result by Lee \& Carney (2002). We propose that contributions from different masses of the Type II suprenovae progenitors that enriched proto-globular clusters' clouds are responsible for this gradient.
\end{abstract}

Keywords. Globular clusters, stars: abundance

\section{Observations and data analysis}

We obtained high signal-to-noise ratio echelle spectra of red giants in 9 old halo clusters using the CTIO $4 \mathrm{~m}$ telescope and its Cassegrain echelle spectrograph. Each spectrum had an effective resolving power of $\mathrm{R}=28,000$ and had complete spectral coverage from 5500 to $7800 \AA$.

The abundance analysis was performed using the Kuruczs plane-parallel LTE model atmospheres and the current version of the LTE line analysis program MOOG.

\section{Results and discussion}

We show our results in Table 1 and Figure 1. The mean $\alpha$-element abundances of our program clusters are in good agreement with other globular clusters, confirming the previous results (Carney 1996). However, [Si/Ti] ratios appear to be anti-correlated with Galactocentric distance. As discussed by Lee et al. (2001) the inner halo globular cluster have the same age as the oldest globular clusters in the outer halo in our Galaxy and the inner parts of the Galaxy most likely formed in high density environments while outer parts in lower density regions. The density of the environment in which clusters formed may affect the initial mass function of Type II supernovae (SNe II) progenitors and the depth of potential in which a SNe II explodes will also affect the abundance ratios. The chemical enrichment by the massive SNe II explosion may lead to elemental abundance anomalies, in the sense that $[\mathrm{Si} / \mathrm{Fe}]$ ratio will increase while $[\mathrm{Ti} / \mathrm{Fe}]$ ratio will decrease (Lee \& Carney 2002). We propose that contributions from different masses of the SNe II progenitors that enriched proto-globular cluster clouds elemental abundances and the different initial physical environments surrounding the proto-globular cluster clouds are responsible for this gradient in $[\mathrm{Si} / \mathrm{Ti}]$ ratios versus Galactocentric distances of the old halo globular clusters. 
Table 1. $[\mathrm{Si} / \mathrm{Ti}]$ ratios of our program clusters.

\begin{tabular}{llllr}
\hline Name & {$[\mathrm{Fe} / \mathrm{H}]$} & {$[\alpha / \mathrm{Fe}]$} & {$[\mathrm{Si} / \mathrm{Ti}]$} & $\begin{array}{r}R_{G C} \\
(\mathrm{kpc})\end{array}$ \\
\hline NGC 288 & -1.51 & +0.44 & +0.22 & 11.6 \\
NGC 5986 & -1.75 & +0.26 & +0.10 & 4.8 \\
NGC 6287 & -2.01 & +0.30 & +0.42 & 1.7 \\
NGC 6293 & -1.99 & +0.32 & +0.50 & 1.4 \\
NGC 6333 & -1.72 & +0.29 & +0.40 & 1.7 \\
NGC 6397 & -1.95 & +0.37 & +0.50 & 6.0 \\
NGC 6541 & -1.76 & +0.36 & +0.31 & 2.2 \\
NGC 6723 & -1.04 & +0.33 & +0.40 & 2.6 \\
NGC 7089 & -1.41 & +0.26 & +0.17 & 10.4 \\
\hline
\end{tabular}

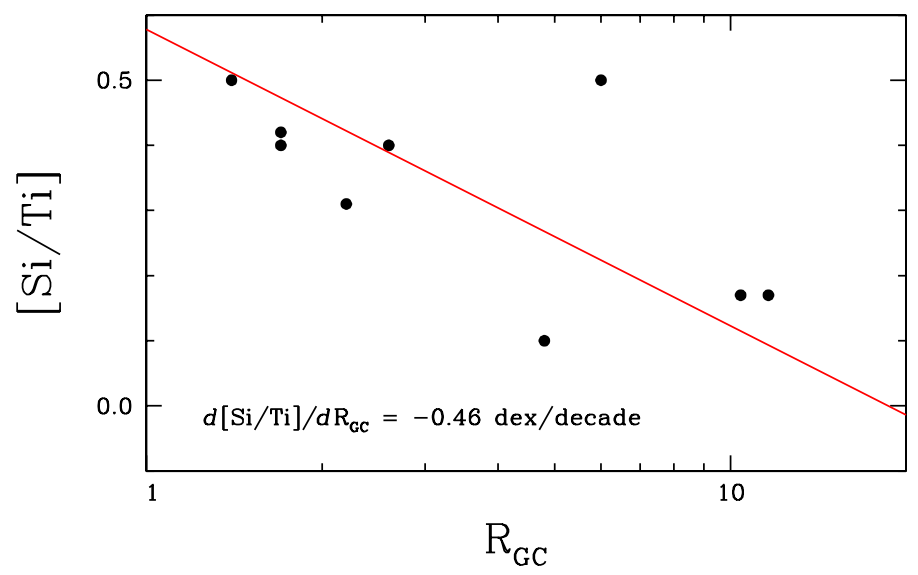

Figure 1. The $[\mathrm{Si} / \mathrm{Ti}]$ ratios of the 9 old halo globular clusters as a function of Galactocentric distance $R_{G C}$. The solid line represents the bisector fit to the data. Our slope of the fit to the data is $\delta[\mathrm{Si} / \mathrm{Ti}] / \delta \log R_{G C}=-0.46$ dex per decade. A non-parametric Spearman rank-order test indicates a probability of $7 \%$ that the anti-correlation between [Si/Ti] and $R_{G C}$ of the 9 old halo globular clusters is random.

\section{Acknowledgements}

Support for this work was provided by the Korea Science and Engineering Foundation (KOSEF) to the Astrophysical Research Center for the Structure and Evolution of the Cosmos (ARCSEC).

\section{References}

Carney, B.W. 1996, PASP 108, 900

Lee, J.-W. \& Carney, B.W. 2002, AJ 124,1511

Lee, J.-W., Carney, B.W., Fullton, L.K., \& Stetson, P.B. 2001, AJ 124, 3136 